Article

\title{
Simplified Reflection Fabry-Perot Method for Determination of Electro-Optic Coefficients of Poled Polymer Thin Films
}

\author{
Dong Hun Park ${ }^{1, *}$, Jingdong Luo ${ }^{2}$, Alex K.-Y. Jen ${ }^{2}$ and Warren N. Herman ${ }^{1}$ \\ 1 Laboratory for Physical Sciences, University of Maryland, College Park, MD 20740, USA; \\ E-Mail: herman@lps.umd.edu (W.N.H.) \\ 2 Department of Materials Sciences and Engineering, University of Washington, Seattle, WA 98198, \\ USA; E-Mails: jdluo@uw.edu (J.L.); ajen@uw.edu (A.K.-Y.J.) \\ * Author to whom correspondence should be addressed; E-Mail: leomac@lps.umd.edu; \\ Tel.: +1-301-935-6431; Fax: +1-301-935-6723.
}

Received: 15 July 2011; in revised form: 12 August 2011 / Accepted: 16 August 2011 / Published: 18 August 2011

\begin{abstract}
We report a simplified reflection mode Fabry-Perot interferometry method for determination of electro-optic (EO) coefficients of poled polymer thin films. Rather than fitting the detailed shape of the Fabry-Perot resonance curve, our simplification involves a technique to experimentally determine the voltage-induced shift in the angular position of the resonance minimum. Rigorous analysis based on optical properties of individual layers of the multilayer structure is not necessary in the data analysis. Although angle scans are involved, the experimental setup does not require a $\theta-2 \theta$ rotation stage and the simplified analysis is an advantage for polymer synthetic efforts requiring quick and reliable screening of new materials. Numerical and experimental results show that our proposed method can determine EO coefficients to within an error of $\sim 8 \%$ if poled values for the refractive indices are used.
\end{abstract}

Keywords: electro-optic polymer; characterization; Fabry-Perot; nonlinear optics

\section{Introduction}

Second-order nonlinear polymers have been widely studied because of their potential applications in high-speed communication and signal processing [1]. A simple and reliable characterization method 
for linear electro-optic (EO) coefficients has been an important issue because researchers who are extensively working on the development of new materials prefer a simple method to quickly screen newly-engineered nonlinear optical (NLO) polymers. Measurements of the EO coefficients of second-order NLO polymers (NLOP) have often been carried out using a reflective arrangement introduced by Teng and Man as well as by Schildkraut, both in 1990 [2,3]. However, in many cases, large systematic errors can result from the simple analysis because it ignores potential multiple reflection effects in a stratified structure [4,5]. In 2006, Park et al. reported a rigorous analysis of the Teng-Man technique taking into account all layers of the sample structure to get a reliable result [4]. This analysis requires accurate layer information, such as anisotropic refractive indices and thickness of each layer. Although single angle of incidence data and analysis can be performed, more extensive data taking at multiple angles together with a fitting process gives the most reliable results.

Using a reflective sample structure as one of the mirrors, Mach-Zehnder interferometry [6,7] and a Michelson interferometry method [8] have been reported for measuring EO coefficients as well as piezoelectric contributions $[7,8]$. They can also be shown to have similar systematic errors if multiple reflection effects are ignored. In addition, these interferometric methods require careful aligning of the signal and reference beams and the interference pattern is susceptible to drift. Although the Mach-Zehnder method can be used to determine both $r_{13}$ and $r_{33}$, the Michelson method can only measure $r_{13}$ and a separate experimental technique must be used to get $r_{33}$. The modified attenuated total reflection (ATR) method $[9,10]$ can also measure $r_{13}$ and $r_{33}$ separately, without an assumption for the value of the ratio $r_{13} / r_{33}$ as required in both the simple and rigorous Teng-Man reflection methods [2-4]. The metal electrode must be removed to enable ATR characterization in order to couple light into the nonlinear thin film using a prism. Removal of the electrode, however, eliminates the option to re-pole the sample under different poling conditions in the process of optimization.

A spectroscopic Fabry-Perot (FP) method was introduced by Uchiki et al. [11] for the characterization of both second- and third-order nonlinearities. This method requires inclusion of a complete picture of the multilayer structure parameters in the analysis. Eldering et al. [12] demonstrated the use of a transmission mode FP etalon with angle scanning at a fixed wavelength to study second-order nonlinear properties of polymer materials by fitting numerical data based on a relatively simple analysis of the multilayer structure requiring two semitransparent electrodes. O'Brien et al. [13] introduced a variation of the transmission FP technique using a thick glass substrate. The use of FP analysis was later applied by Pretre et al. [14] to decal-deposited reflection FP structures in order to measure complex linear optical and electro-optic parameters as well as to characterize the inverse piezoelectric contribution. This analysis takes into account the complete multilayer structure properties and requires fitting the detailed shape of the Fabry-Perot resonance curve. This reflective FP method also requires a $\theta-2 \theta$ rotation stage in the experimental setup.

In this paper, we present a somewhat simplified experimental setup not requiring a $\theta-2 \theta$ rotation stage together with a simplified analysis of reflection mode Fabry-Perot interferometry. As in the rigorous FP analysis methods [14], a transparent yet highly reflective layer such as a transparent conducting oxide (TCO) or thin gold is employed. In this simplified technique, however, the analysis of data does not require separate measurement and fitting of the optical properties of the electrodes, as is required in earlier [14] rigorous FP methods. Rather than fitting the detailed shape of the Fabry-Perot resonance curve as in [14], our simplification involves a technique to experimentally 
determine the voltage-induced shift in the angular position of the resonance minimum. The simplified method can give a reliable measurement within an error of $\sim 8 \%$ at telecommunication wavelengths of 1,300 $\mathrm{nm}$ and $1,550 \mathrm{~nm}$ without including the complete multilayer structure in the analysis. We show numerical simulations using rigorous analysis to justify the simplified analysis for extracting EO coefficients. Experimental results are given and we discuss limitations and errors depending on optical properties of the electrode layers and nonlinear films.

\section{Experimental Setup and Data Analysis}

Angle-dependent reflectivity and voltage-modulated reflectivity data are collected using the experimental setup shown in Figure 1. A polarizer and half wave plate are used to produce the two independent linearly polarized states. A large area infrared photo-detector is used to detect reflected light over a wide range of angles of incidence in order to collect angular data without the need for the $\theta-2 \theta$ rotation stage used in [14] to maintain alignment as the laser source arm rotates.

Figure 1. Schematic of Fabry-Perot (FP) experimental setup (left) and representative reflectivity $R$ and modulated reflectivity $\Delta R$ from digital voltmeter (DVM) and lock-in amplifier, respectively (right). $\mathrm{P}, \mathrm{HP}, \mathrm{PD}$, and $\mathrm{AC}$ are polarizer, half wave plate, photo-detector, and voltage source, respectively. NLOP: nonlinear optical polymers

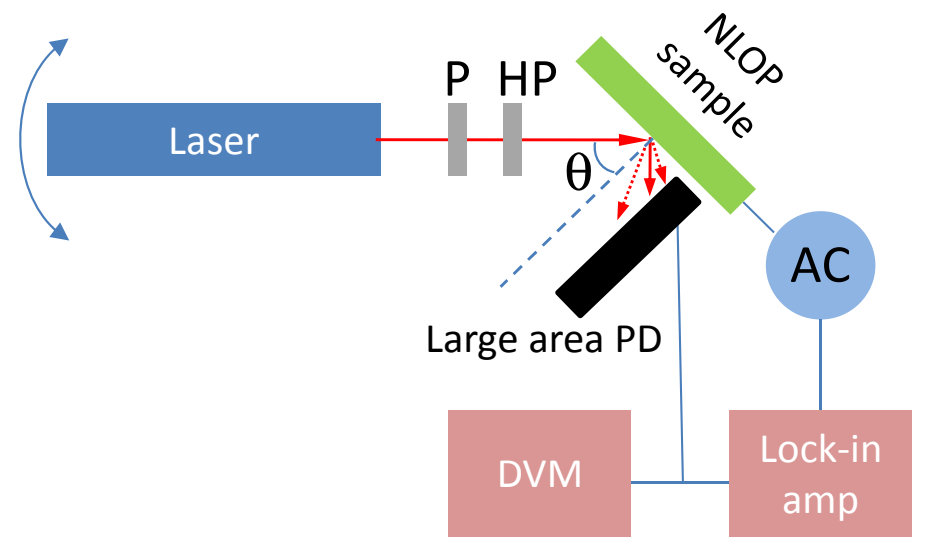

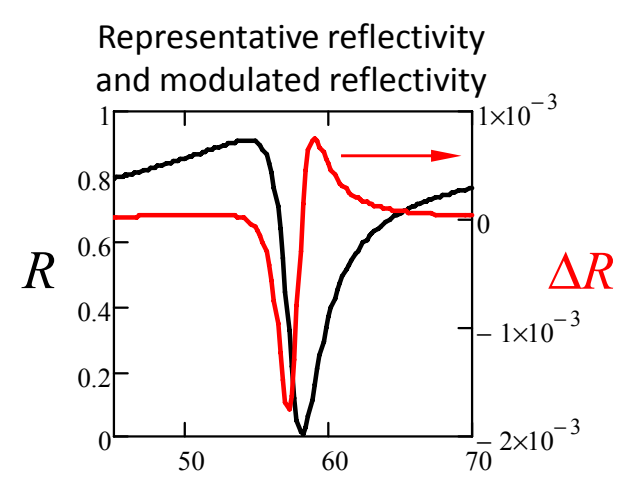

Angle of incidence

In order to analyze the experimental data for determination of the two independent EO coefficients $r_{13}$ and $r_{33}$ of poled polymer thin films, we consider the phase condition for reflection minima for two different FP resonator structures shown in Figure 2: (a) the idealized simple three layer structure (air/NLOP/air) and (b) the multilayer structure (glass/TCO/NLOP/metal). We first use the mathematical expression for reflectivity to discuss the phase condition from the simple three layer structure, which can be found in most optics textbooks [15]. Then we revisit the rigorous expression for reflection from a multilayer structure, which has already been described previously [11,14], for application of the phase condition to the practical multilayer structure and for justification of our simplified analysis. 
Figure 2. Schematics of two Fabry-Perot structures: (a) An ideal simple three-layer structure (air/NLOP/air); (b) a practical four-layer structure (glass/TCO/NLOP/metal).

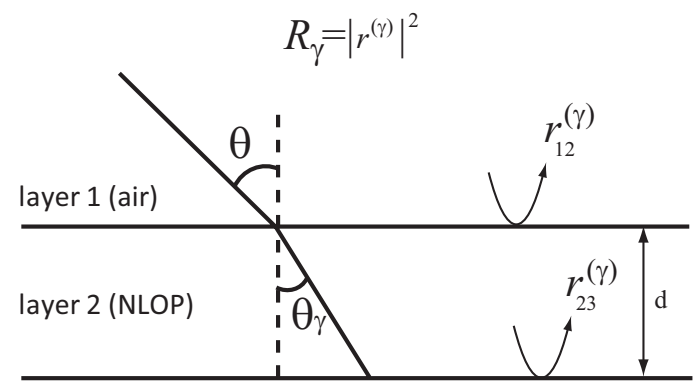

layer 3 (air)

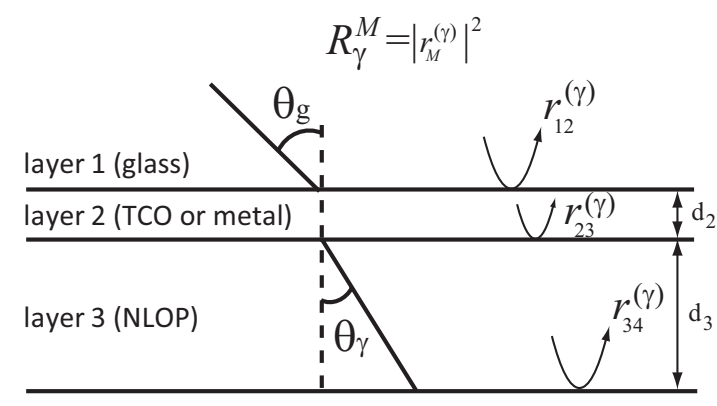

layer 4 (bottom metal layer)

(b)

(a)

\subsection{Simplified Analysis Based on Ideal Three Layer Fabry-Perot Structure}

The reflection coefficient for the simple three-layer FP resonator can be obtained using Airy's formula [15]. It is well known that the intensity of a reflected wave is a periodic function with respect to the phase of the reflected wave. In the case that the film is lossless, the reflectivity is given by

$$
R_{\gamma}=\left|\frac{r_{12}^{(\gamma)}+r_{23}^{(\gamma)} e^{i 2 \psi^{(\gamma)}}}{1+r_{12}^{(\gamma)} r_{23}^{(\gamma)} e^{i 2 \psi^{(\gamma)}}}\right|^{2}=\frac{4\left|r_{12}^{(\gamma)}\right|^{2} \sin ^{2}\left(\psi^{(\gamma)}\right)}{\left(1-\left|r_{12}^{(\gamma)}\right|^{2}\right)^{2}+4\left|r_{12}^{(\gamma)}\right|^{2} \sin ^{2}\left(\psi^{(\gamma)}\right)}
$$

where $\gamma=s$ or $p$ represents transverse electric (TE) and transverse magnetic (TM) polarizations, respectively. The reflection coefficient at a single interface from $j$-layer to $k$-layer is

$$
r_{j k}^{(\gamma)}=\frac{Z_{k}^{(\gamma)}-Z_{j}^{(\gamma)}}{Z_{k}^{(\gamma)}+Z_{j}^{(\gamma)}}
$$

with the $s$ - and $p$ - wave impedance of each layer given by

$$
Z^{(s)}=\frac{1}{\sqrt{n_{o}^{2}-\sin ^{2} \theta}}, \quad Z^{(p)}=\frac{1}{n_{o}} \sqrt{1-\left(\frac{\sin \theta}{n_{e}}\right)^{2}}
$$

where $\theta$ is the exterior angle of incidence from air and $n_{\mathrm{o}}$ and $n_{\mathrm{e}}$ are ordinary and extraordinary indices of refraction of the nonlinear poled organic thin film belonging to the point group symmetry $\infty m m$ (space group $C_{\infty v}$ ). The phase terms for $s$ - and $p$ - polarized light are

$$
\psi^{(\gamma)}=k_{o} n_{\gamma} \cos \theta_{\gamma} d
$$

where $k_{o}(=\lambda / 2 \pi)$ is the wave vector in vacuum, $\lambda$ is the optical wavelength and $d$ is the thickness of the film. Note that the reflectivity minima occur only when $\psi^{(\gamma)}=m \pi, m=0,1,2, \cdots$. When an external electric field is applied to the nonlinear film, the refractive index and the internal angle $\theta_{\gamma}$ change simultaneously. In terms of the exterior angle of incidence $\theta$, Equation (4) can be written

$$
\psi^{(\gamma)}=k_{o} d \sqrt{n_{\gamma}^{2}-\sin ^{2} \theta}
$$

having used Snell's law. Because the phase described by Equation (5) has a fixed value $(m \pi)$ at the reflectivity minima, we can derive the angular shift of the reflection minima produced by the change of 
refractive index. Ignoring inverse piezoelectric effects, the expression for the derivative of $n_{s}\left(=n_{o}\right)$ and of $n_{p}$ with respect to the exterior angle of incidence $\theta$ is

$$
\frac{\partial n_{\gamma}}{\partial \theta}=\frac{\sin 2 \theta}{2 n_{\gamma}} \approx \frac{\Delta n_{\gamma}}{\Delta \theta}
$$

obtained from Equation (5) by setting the total differential of the phase equal to zero (i.e., $d \psi_{\gamma}=0$ ). The corresponding expression including the inverse piezoelectric effect is given in the Appendix.

From the angular shift $\Delta \theta$ of reflectivity minima, the modulated refractive indices $\Delta n_{s}$ and $\Delta n_{p}$ can be calculated using Equation (6). $\Delta n_{o}=\Delta n_{s}$ is then obtained directly. $\Delta n_{e}$ is obtained from $\Delta n_{p}$ and $\Delta n_{o}$ using the index ellipsoid for a uniaxial anisotropic medium [15], viz.,

$$
\frac{1}{n_{p}\left(\theta_{p}\right)^{2}}=\frac{\cos ^{2} \theta_{p}}{n_{o}^{2}}+\frac{\sin ^{2} \theta_{p}}{n_{e}^{2}} \Rightarrow n_{p}(\theta)^{2}=n_{o}^{2}\left(1-\frac{\sin ^{2} \theta}{n_{e}^{2}}\right)+\sin ^{2} \theta
$$

By differentiation we get

$$
\Delta n_{p}=\frac{1}{n_{p}(\theta)}\left[n_{o}\left(1-\frac{\sin ^{2} \theta}{n_{e}^{2}}\right) \Delta n_{o}+\frac{\left(n_{o} \sin \theta\right)^{2}}{n_{e}^{3}} \Delta n_{e}\right]
$$

which includes the effect of the varying angle of refraction inside the polymer under applied voltage. Finally, solving for $\Delta n_{e}$ gives

$$
\Delta n_{e}=\frac{n_{e}^{3}}{\left(n_{o} \sin \theta\right)^{2}}\left[n_{p}(\theta) \Delta n_{p}+n_{o}\left(\frac{\sin ^{2} \theta}{n_{e}^{2}}-1\right) \Delta n_{o}\right]
$$

It should be noted that Equation (8) is somewhat different from the corresponding Equation (29) in [12], where the change of refraction angle inside the polymer was ignored.

Experimentally, direct observation of the angular shift of reflectivity minima under an applied voltage $V$ is not easy because of the small angular shift and uncertainty of minima position. Application of a high voltage for direct observation may lead to damage on the thin film and the metal electrode. Use of a lock-in amplifier permits measurement of small modulations, but it is impossible to detect modulation at the reflection minima because the slope of the reflectivity is zero at the minima. Figure 3 shows representative reflectivity curves with and without an applied voltage. Assuming the new reflectivity curve $R(V)$ under the external voltage $V$ is only shifted slightly in $\theta$ from $R(V=0)$, by expanding to 1 st order we have

$$
\Delta \theta=\frac{\Delta R_{\gamma}}{\frac{\partial R_{\gamma}}{\partial \theta}}
$$

The modulated reflectivity $\Delta R_{\gamma}$ is measured by lock-in amplifier at incident angles around the reflectivity minima where the voltage-modulated reflectivity is in the linear regime for validation of a Taylor expansion to first order. These angles can be found where the reflectivity has the largest gradient, viz., inflection points. The derivative $\partial R_{\gamma} / \partial \theta$ is numerically calculated from experimental reflectivity data as a function of angle of incidence. The changes of refractive index $\Delta n_{o}$ and $\Delta n_{p}$ are calculated using 


$$
\Delta n_{\gamma}=\frac{\partial n_{\gamma}}{\partial \theta} \Delta \theta \approx \frac{\sin 2 \theta}{2 n_{\gamma}} \cdot \frac{\Delta R_{\gamma}^{M}}{\frac{\partial R_{\gamma}^{M}}{\partial \theta}}
$$

based on Equations (6) and (10). Finally the EO coefficients $r_{13}$ and $r_{33}$ are obtained using

$$
\Delta n_{o}=-\frac{1}{2} n_{o}^{3} r_{13} \frac{V}{d_{3}}, \quad \Delta n_{e}=-\frac{1}{2} n_{e}^{3} r_{33} \frac{V}{d_{3}}
$$

where $V$ is the peak applied voltage to the film, and $n_{o}, n_{e}$, and $d_{3}$ are the separately measured anisotropic indices and thickness of the NLOP film.

Figure 3. Representative reflective curves without bias $R(V=0)$ and with bias $R(V)$ to the film as a function of angle of incidence. $\Delta R$ can be measured using a lock-in amplifier and the slope $\partial R / \partial \theta$ can be obtained by numerical differentiation of the experimental reflectivity curve.

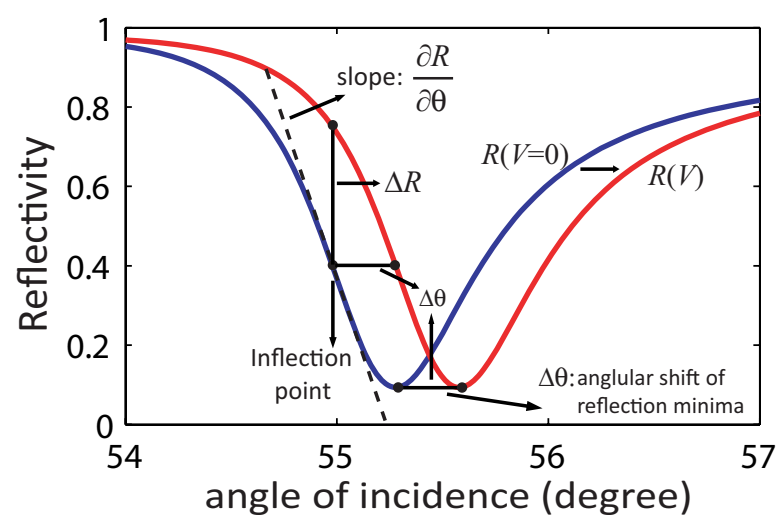

Equations (9), (11), and (12) are all that is needed to determine $r_{13}$ and $r_{33}$ in this simplified model. In the next section, we explore the validity of using Equation (6) for the practical multilayer structure of Figure 2(b). It should again be noted that Equation (6) holds only at the reflectivity minima. Therefore, the angle at the inflection point should be close enough to the reflectivity minima in order to minimize a possible systematic error. Such a sharp reflectivity curve can be obtained from a high Q resonator structure containing a highly reflective transparent electrode layer. A transmission type FP method with two transparent conducting electrodes can be used, but the low finesse resulting from the relatively low reflectivity would cause broadening of the transmission peak [14].

\subsection{Rigorous Analysis Justification of the Simplified Method for a Practical FP Structure}

A practical poled polymer thin film sample is a multilayer structure glass/(TCO or metal)/NLOP/metal as shown in Figure 2(b). Similar to the simple FP structure, the reflection coefficient of the multilayer structure has the form [15]

$$
r_{M}^{(\gamma)}=\frac{r_{12}^{(\gamma)}+\hat{r}_{23}^{(\gamma)} e^{i 2 \psi_{2}^{(\gamma)}}}{1+r_{12}^{(\gamma)} \hat{r}_{23}^{(\gamma)} e^{i 2 \psi_{2}^{(\gamma)}}} \Leftarrow \hat{r}_{23}^{(\gamma)}=\frac{r_{23}^{(\gamma)}+r_{34}^{(\gamma)} e^{i 2 \psi_{3}^{(\gamma)}}}{1+r_{23}^{(\gamma)} r_{34}^{(\gamma)} e^{i 2 \psi_{3}^{(\gamma)}}}
$$

where $\psi_{j}^{(\gamma)}$ is the phase of $\gamma$-polarized light at the $j$-layer as shown in Figure 2(b) and can be calculated using Equation (5) with corresponding refractive indices and thickness. Care must be taken 
to note that the layer labels corresponding to each layer in Figure 2(b) are different from those in Figure 2(a). However, the reflection coefficient $r_{k}^{(\gamma)}$ can be calculated using Equation (2) with corresponding refractive indices and thickness. The reflectivity for this multilayer structure is

$$
R_{\gamma}^{M}=\left|r_{M}^{(\gamma)}\right|^{2}=\left|\frac{r_{12}^{(\gamma)}+\hat{r}_{23}^{(\gamma)} e^{i 2 \psi_{2}^{(\gamma)}}}{1+r_{12}^{(\gamma)} \hat{r}_{23}^{(\gamma)} e^{i 2 \psi_{2}^{(\gamma)}}}\right|^{2}
$$

but the explicit form of the phase condition for reflectivity minima is quite complicated. Numerical simulation, however, shows that the angular shift of the reflectivity minima resulting from the change of refractive index can still be calculated from Equation (6), which indicates that the value of the phase $\psi^{(\gamma)}$ at the reflectivity minima can still be expressed as $\psi^{(\gamma)}=m \cdot \pi+$ constant, where, at least to first order, "constant" is independent of the refractive index and angle of incidence as is in the previous simple FP case.

Figure 4 shows the simulated errors of $r_{13}$ and $r_{33}$ with varying wavelengths of $1,300 \mathrm{~nm}-1,550 \mathrm{~nm}$ for one of the many parameter combinations we explored We assumed a $4 \mu \mathrm{m}$ thick NLOP film with dispersive anisotropic indices and EO coefficients $\left(n_{\mathrm{o}}=1.73, n_{\mathrm{e}}=1.75, r_{13}=50 \mathrm{pm} / \mathrm{V}\right.$, and $r_{33}=150 \mathrm{pm} / \mathrm{V}$ at $1,550 \mathrm{~nm}$ ) on $30 \mathrm{~nm}$ thick gold layer and calculated the angular shift of the reflection minima obtained from Equation (14) under the application of $5 \mathrm{~V}$ to the film. Then, Equations (9), (11), and (12) were used to extract values for EO coefficients from the changes of anisotropic refractive indices and subsequently compared with the input EO coefficients to obtain the error estimate as shown in Figure 4. The overall errors of both $r_{13}$ and $r_{33}$ in the telecommunication wavelength range fall under $\sim 3 \%$, indicating that Equation (6) is valid for the analysis of the multilayer FP structure shown in Figure 2(b). It should be noted that we use Equation (14) for numerical modeling to verify the proposed method, but not for data analysis.

Figure 4. Simulated errors of $r_{13}$ (blue) and $r_{33}$ (red) with varying wavelengths from the calculated angular shift of the reflection minima.

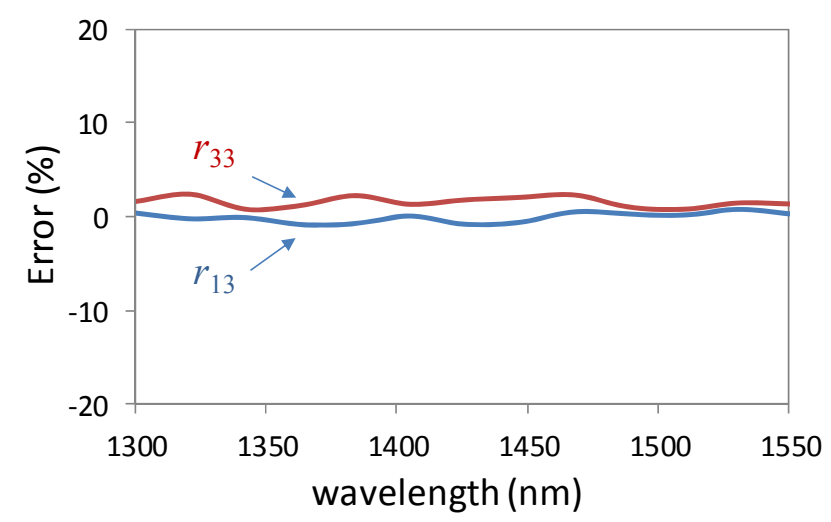

In principle, one should multiply the reflection coefficient in Equation (13) by the transmission coefficients at the air/glass and glass/air interfaces, but these do not contribute to the calculation of $\Delta \theta$ because they cancel out in Equation (10). Also, angle-dependent transmission at the detector facet is canceled out when the calculation of Equation (10) is performed.

Error in the EO coefficients can also result from the uncertainty in the film thickness and anisotropic indices, $n_{o}$ and $n_{e}$. This error is directly proportional to the uncertainty in the film thickness 
while the uncertainty in the refractive indices produces about three times higher errors in the EO coefficients because of the cubic dependence indicated in Equation (12). For example, assuming that an unpoled refractive index is 1.71 and the birefringence after the poling process is 0.03 with $n_{o}=1.7$ and $n_{e}=1.73$, one might simply use the unpoled index for the analysis, which makes $0.6 \%$ and $1.2 \%$ errors in $n_{o}$ and $n_{e}$, respectively; then the errors in $r_{13}$ and $r_{33}$ caused only by the uncertainty in refractive indices are about $1.8 \%$ and $3.5 \%$, respectively. In order to minimize these errors, the film thickness and anisotropic indices should be measured more precisely by prism coupling or other techniques, such as ellipsometry, after collection of the reflection FP data and removal of the metal layer. Although removal of the metal layer is also required for the ATR measurement technique, the data analysis is more straightforward in our simplified FP method.

\section{Numerical Simulations to Determine Range of Validity}

We investigated the suitability of both ITO and a thin gold layer as the transparent electrode to determine which type of electrode is appropriate for reliable estimation of EO coefficients. Numerical simulation has been performed based on the equations described in Section 2. We simulated three different cases using $30 \mathrm{~nm}$ thick gold and two different ITOs as the transparent electrode. The complex index of refraction, $n$ and $\kappa$, of gold from [16] was used for the numerical calculation. Optical properties of two different ITOs, one manufactured by Delta Technology (DT) and the other by Thin Film Devices (TFD), were measured using a J. A. Woollam ellipsometer VASE ${ }^{\circledR}$ as shown in Figure 5. In the infrared, the linear absorption resulting from the extinction coefficient $\kappa$ of $200 \mathrm{~nm}$ thick DT ITO is seen to be larger than that of $45 \mathrm{~nm}$ thick TFD ITO and it becomes even larger as the wavelength increases.

Figure 5. Complex index of refraction $(n+\mathrm{i} \kappa)$ of two ITOs (black: $200 \mathrm{~nm}$ thick Delta Technology, red: $45 \mathrm{~nm}$ thick Thin Film Devices) measured by ellipsometer. The inset shows a complex index of refraction of gold from [16].

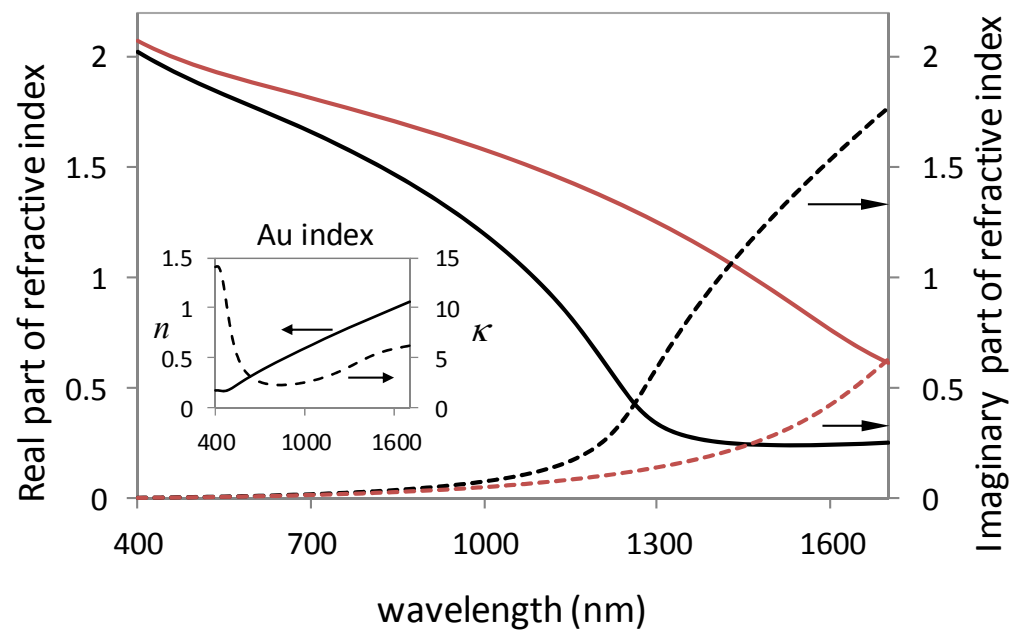

Figure 6(a) shows simulated reflectivities of $p$-polarized light $[15,17]$ from multilayer structures containing either $30 \mathrm{~nm}$ thick gold or one of two ITO's (DT and TFD) as a top transparent electrode between the glass substrate and the NLOP film layer. The bottom metal (gold) layer is assumed to 
be $\sim 3$ times thicker than its skin depth in order to ignore the back reflection from the gold/air interface. The sample containing $30 \mathrm{~nm}$ thick gold shows the highest finesse because of the strong reflection from gold, while the reflectivity dip from the TFD ITO layer is much broader than that of the DT ITO layer because it is relatively thin and has a smaller extinction coefficient as shown in Figure 5. A low Q factor as shown in the sample containing a TFD ITO layer turns out to be inappropriate for the proposed FP method because of the large systematic error resulting from the use of Equation (10) to determine the voltage-induced angular shift of the resonance minimum. The derivative of these reflectivity curves are shown in Figure 6(b). They were numerically calculated from the simulated reflectivity curves.

Figure 6. (a) Simulated reflectivity of p-polarized light from NLO samples containing gold (black), DT (blue), or TFD (red) ITO at the wavelength of 1,550 nm. 1.73 and 1.75 were used for no and ne of NLOP, respectively and the NLOP film thickness was assumed to be $4 \mu \mathrm{m}$; (b) Simulated derivative of reflectivity data as a function of angle of incidence.
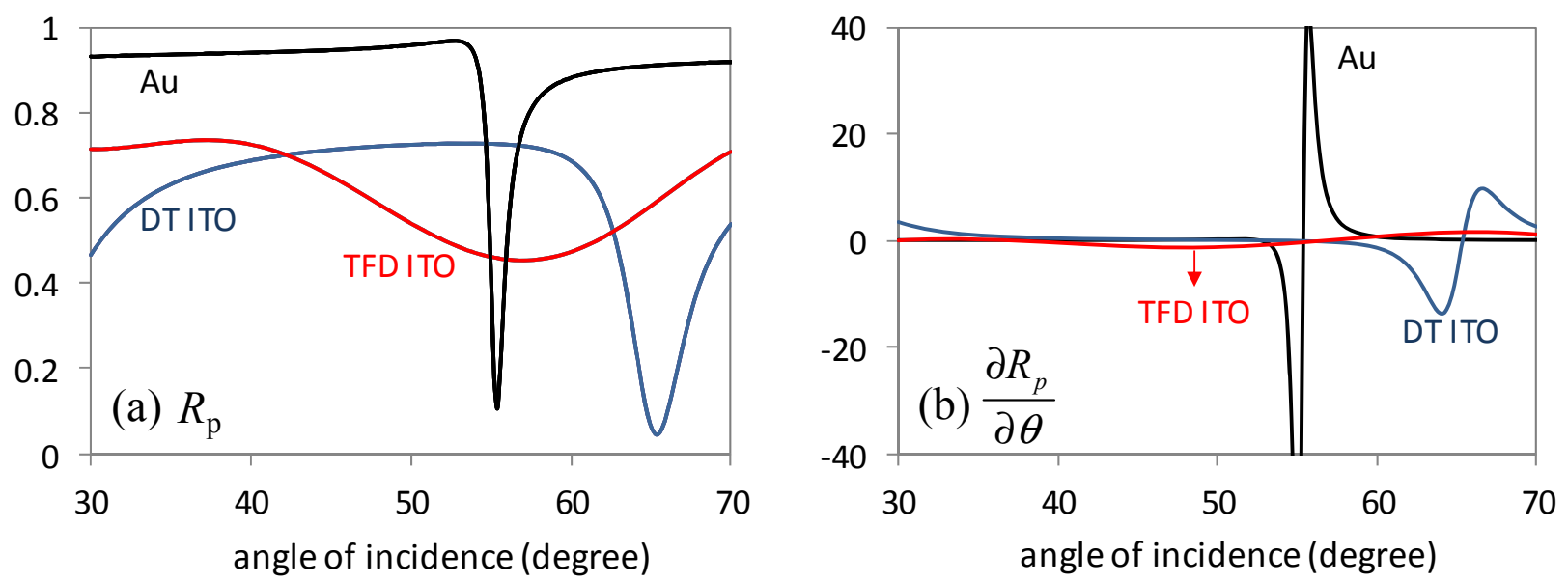

Figure 7 shows simulated $\Delta n_{\mathrm{e}}$ and $\Delta R_{p}$ using Equations (9), (11) and (14) at 1,550 nm when a $30 \mathrm{~nm}$ thick gold layer and a $200 \mathrm{~nm}$ thick DT ITO were used as the transparent electrode. We find for both cases that the modulated refractive index $\Delta n_{\mathrm{e}}$, which is directly proportional to the EO coefficient $r_{33}$, is within about $5 \%$ at the angle of incidence corresponding to the highest reflectivity modulation. Note that higher accuracy (less than $2 \%$ error) was achieved from the sample containing the $30 \mathrm{~nm}$ thick gold layer. Typical indices of refraction for NLOPs range from 1.5 to 2 at telecommunication wavelengths. In this range of refractive index and at the wavelengths of $1,300 \mathrm{~nm}$ and 1,550 nm, numerical simulations indicate that less than $4 \%$ error is expected to be obtained with the $30 \mathrm{~nm}$ gold layer, whereas the DT ITO gave less than $6 \%$ error with varying thickness and refractive index. For $s$-polarized light, the modulated refractive index showed much smaller errors (within $2 \%$ ) compared to p-polarized light. 
Figure 7. Simulated $\Delta n_{\mathrm{e}}$ (solid curves) and $\Delta R_{p}$ (dashed curves) from a sample containing a $30 \mathrm{~nm}$ thick gold (black) and a Delta Technology ITO (blue) under assumption of no errors in anisotropic indices of $n_{\mathrm{o}}$ and $n_{\mathrm{e}}$. Gray region shows $\pm 5 \%$ error bound of $\Delta n_{\mathrm{e}}$, directly corresponding to the error bound of an EO coefficient $r_{33}$.

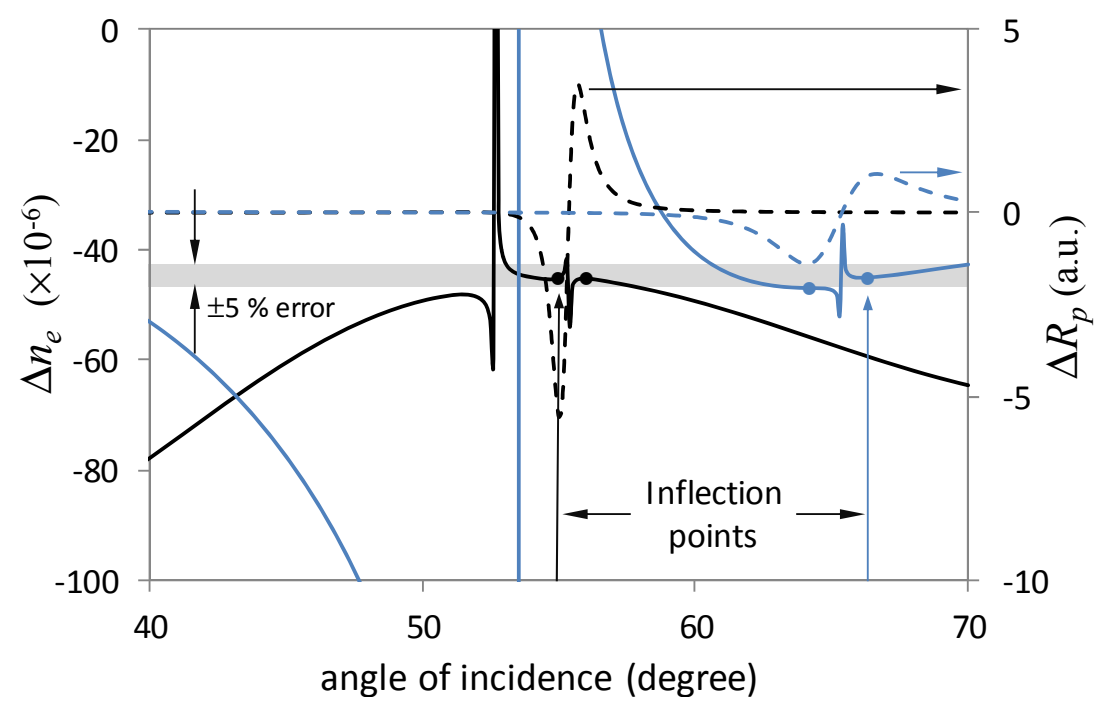

The sample containing the TFD ITO layer, however, may give an error higher than $50 \%$ because of its low finesse resulting from relatively low reflectivity of the TFD ITO. Therefore, careful selection of a transparent conducting oxide should be made in order to make a higher Q resonator structure and reduce the error from using the simplified analysis. Once a highly reflective (absorptive) transparent conducting layer such as DT ITO or thin Au layer is employed in the sample, Equation (11) in the simplified method can be used to measure EO coefficients within an error of $\sim 5 \%$ if the refractive indices, thickness, and angles are known precisely. On the contrary, when the simple analysis is used in other ellipsometric or interferometric methods such as Teng-Man, Mach-Zehnder, and Michelson, multiple reflection effects caused by a highly reflective transparent conducting layer may give a much larger error. We also note that a large linear absorption by the nonlinear film will result in broadening of the reflectivity and lowering the $\mathrm{Q}$ factor, which could affect the accuracy. However, we find that this method is still appropriate even for a highly absorptive film provided $\kappa=$ is not greater than $\sim 0.01$, corresponding to $\sim 3,000 \mathrm{~dB} / \mathrm{cm}$ at telecommunication wavelengths. The electro-chromic effect, the change of the extinction coefficient with applied field, can be ignored in this range of the extinction coefficient $\kappa$. Also, Equation (6) is still valid because only the real part of the phase term in Equation (4) determines the phase condition for the reflection minima.

In this simulation, we ignored any inverse piezoelectric contribution, but it can be characterized as described in the Appendix. According to [7], use of a modulation frequency above $\sim 1 \mathrm{kHz}$ can minimize piezoelectric effects for AJLZ53/APC to $d_{33}<\sim 2 \mathrm{pm} / \mathrm{V}$. A $d_{33}$ less than $\sim 1 \mathrm{pm} / \mathrm{V}$ was reported for the materials investigated in $[8,14]$. However, materials with a relatively large piezoelectric contribution compared to EO effects would require piezoelectric measurements for accurate determination of EO coefficients. It is clear that the uncertainty of this simplified technique strongly depends on the finesse of FP structures and we claimed that highly reflective transparent conducting layer such as DT ITO or thin Au layer can be used for the EO characterization within 5\% error provided the refractive indices, thickness and angular positions are known precisely. An 
uncertainty of $\pm 0.5^{\circ}$ in the angular position of the reflection minimum and $\sim 2 \%$ error in thickness can cause $\sim 1 \%$ and $\sim 2 \%$ errors in the EO coefficients, respectively. Including these uncertainties from angular position and film thickness, the total error of the simplified FP technique is expected to be about $8 \%$. As discussed earlier, using unpoled rather than poled values for the refractive index can typically contribute another $2 \%$ and $4 \%$ error in $r_{13}$ and $r_{33}$, respectively.

For characterization at telecommunication wavelengths, the nonlinear film must have appropriate thickness in order to have a reflectivity dip within a range of angle of incidence from about $30-60^{\circ}$. This is also true for the previous reflective FP method introduced in [14] when using infrared wavelengths. For example, when the film index is about 1.7 at the wavelength of $1,550 \mathrm{~nm}$, a $4-5 \mu \mathrm{m}$ thick film will guarantee a reflectivity dip in this angle of incidence range. A rough rule of thumb is $d_{3}>3.7 \lambda$. Lower refractive index and shorter wavelength allow the NLOP film to be thinner. A thicker film may limit poling efficiency because a higher voltage for maintaining the same poling electric field can lead to damage on the film and/or the electrode. Note that there will also be bands of smaller film thickness $\left(d_{3}<3.7 \lambda\right)$ allowing resonances in the measurable angle of incidence range, but their prediction requires the use of Equation (14).

Figure 8. Existence maps of reflection minima in the angle of incidence range $30-60^{\circ}$ as a function of unpoled index and film thickness using $20 \mathrm{~nm}$ thick gold as the transparent electrode: (a) 1,550 nm with $s$-polarized light; (b) 1,550 nm with $p$-polarized light; (c) 1,320 nm with $s$-polarized light; and (d) 1,320 nm with $p$-polarized light. Black regions indicate the existence of at least one reflection minimum whereas white regions indicate the absence of reflection minima.

(a)
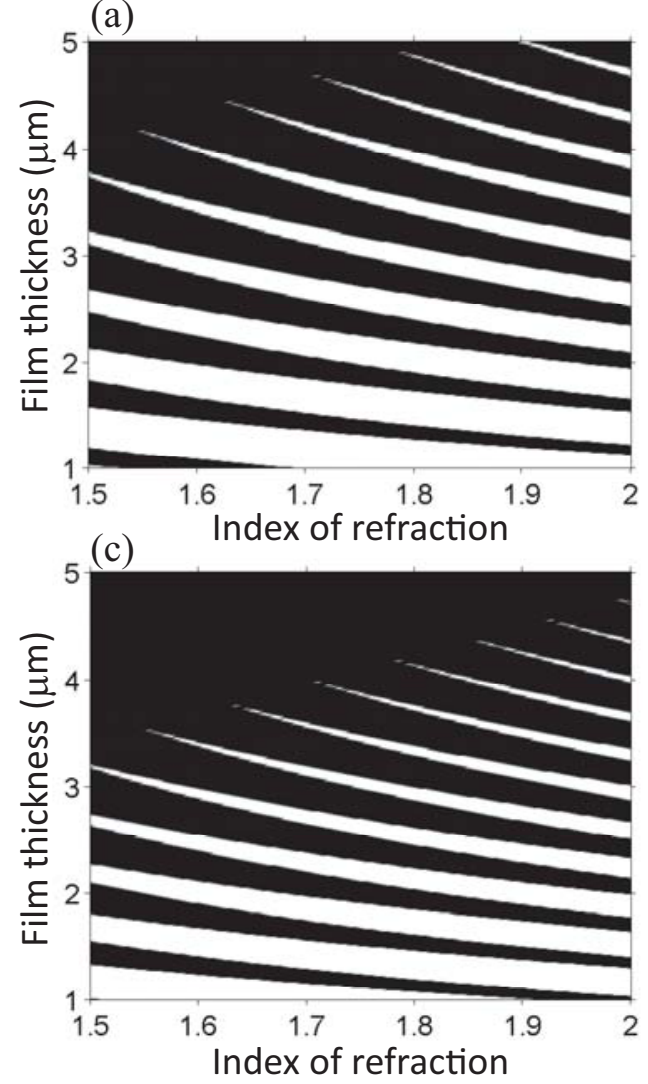

(b)
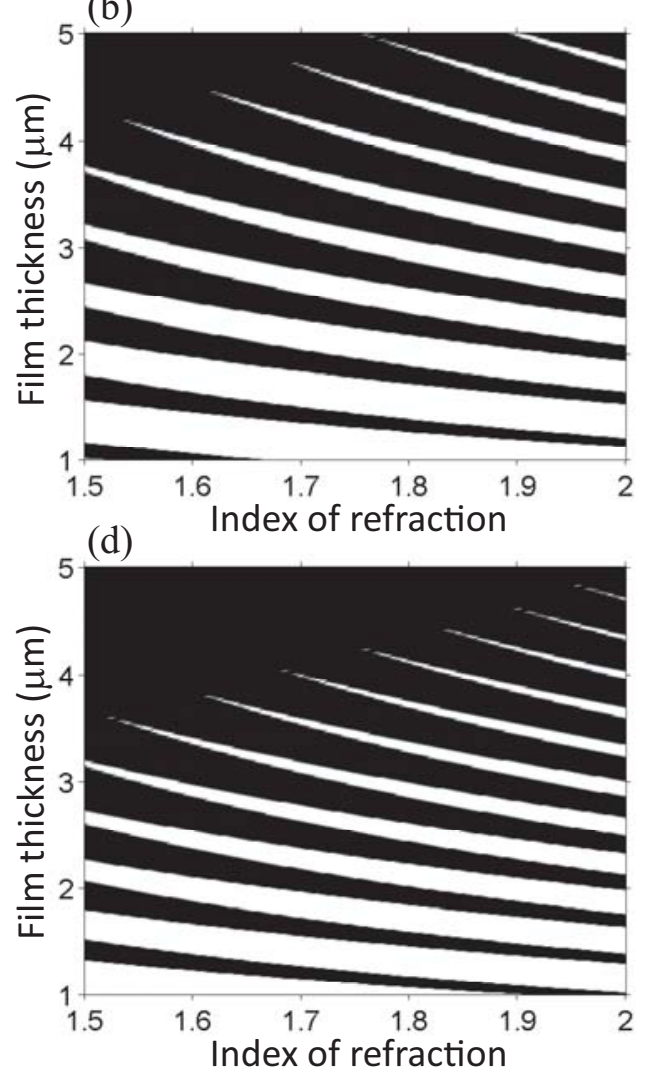
Figure 8 shows existence maps for reflection minima as a function of unpoled refractive index and film thickness within the range of angles of incidence between $30^{\circ}$ and $60^{\circ}$ when $20 \mathrm{~nm}$ thick gold is used as a transparent electrode for wavelengths of 1,320 $\mathrm{nm}$ and 1,550 nm. Increasing the gold thickness to $30 \mathrm{~nm}$ has little effect on the plots. Birefringence $\Delta n\left(=n_{e}-n_{o}\right)$ of the nonlinear film will slightly shift the reflection minima, but $\Delta n$ of less than $\sim 0.06$ will not affect the maps very much. When birefringence is large, on the other hand, one might need to extend the range of angles to capture a reflection minimum. For wavelengths other than 1,320 nm and 1,550 nm, Equation (14) can be applied to predict the angular position of the reflection minima to find the appropriate film thickness.

\section{Experimental Example}

A nonlinear polymer thin film was prepared with 30 wt.\% of AJLZ53, in an amorphous polycarbonate (APC) host as shown in Figure 9. The $10 \mathrm{wt} . \%$ doped polymer (AJLZ53 + APC) was dissolved in dibromomethane for a few hours. The solution was filtered through $0.2 \mu \mathrm{m}$ poly(tetrafluoroethylene) (PTFE) filters and spin-coated to produce a $\sim 5 \mu \mathrm{m}$ thick film on $200 \mathrm{~nm}$ thick indium tin oxide (ITO)/glass substrate manufactured by Delta Technologies (Product \#: CB-40IN-0111). The film was dried under vacuum overnight at $80{ }^{\circ} \mathrm{C}$ to remove residual solvent. A $100 \mathrm{~nm}$ thick gold layer was deposited on the film using an electron-beam evaporator (CHA Mark-40) to serve as a poling electrode and reflector. Poling was performed in an inert nitrogen gas atmosphere near the glass transition temperature of $135{ }^{\circ} \mathrm{C}$. The sample was heated up at $10{ }^{\circ} \mathrm{C} / \mathrm{min}$ and the voltage was slowly increased to $\sim 50 \mathrm{~V} / \mu \mathrm{m}$. Once the temperature of $135{ }^{\circ} \mathrm{C}$ was reached, the sample was cooled down to room temperature rapidly with the voltage on.

Figure 9. Molecular structures of AJLZ53 chromophore (left) and polycarbonate (right).
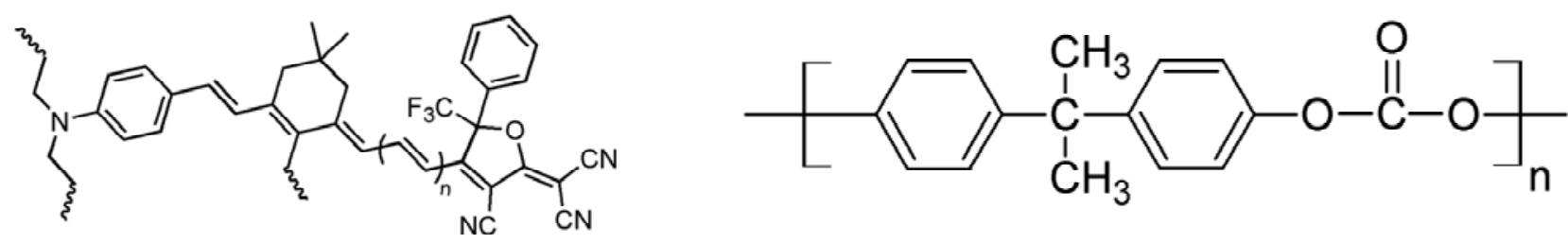

Figure 10 shows the experimental data from 30 wt.\% AJLZ53/APC. Because we employed highly reflective DT ITO in this sample, the simplified method was used to estimate EO coefficients. The procedure is as follows. (i) Measure the reflectivity curves $R_{\gamma}^{M}$ using a DC voltmeter and the modulated reflectivity curves $\Delta R_{\gamma}^{M}$ using a lock-in amplifier as a function of angle of incidence for s- and p- polarized light as shown in Figure 10; (ii) Numerically calculate the derivatives of each experimental reflectivity curve $\partial R_{\gamma}^{M} / \partial \theta$; (iii) Calculate the angular shift of the reflection minimum using Equation (10) applied at the maximum modulation point (inflection point); (iv) Calculate the voltage-induced changes in refractive indices and finally the EO coefficients using Equations (11) and (12), respectively, with known film thickness and anisotropic indices of refraction. 
Figure 10. Experimental results from AJLZ53/APC. The measured reflectivities (Rs and Rp: left axis) and modulated reflectivities (Rs and Rp: right axis) as a function of angle.
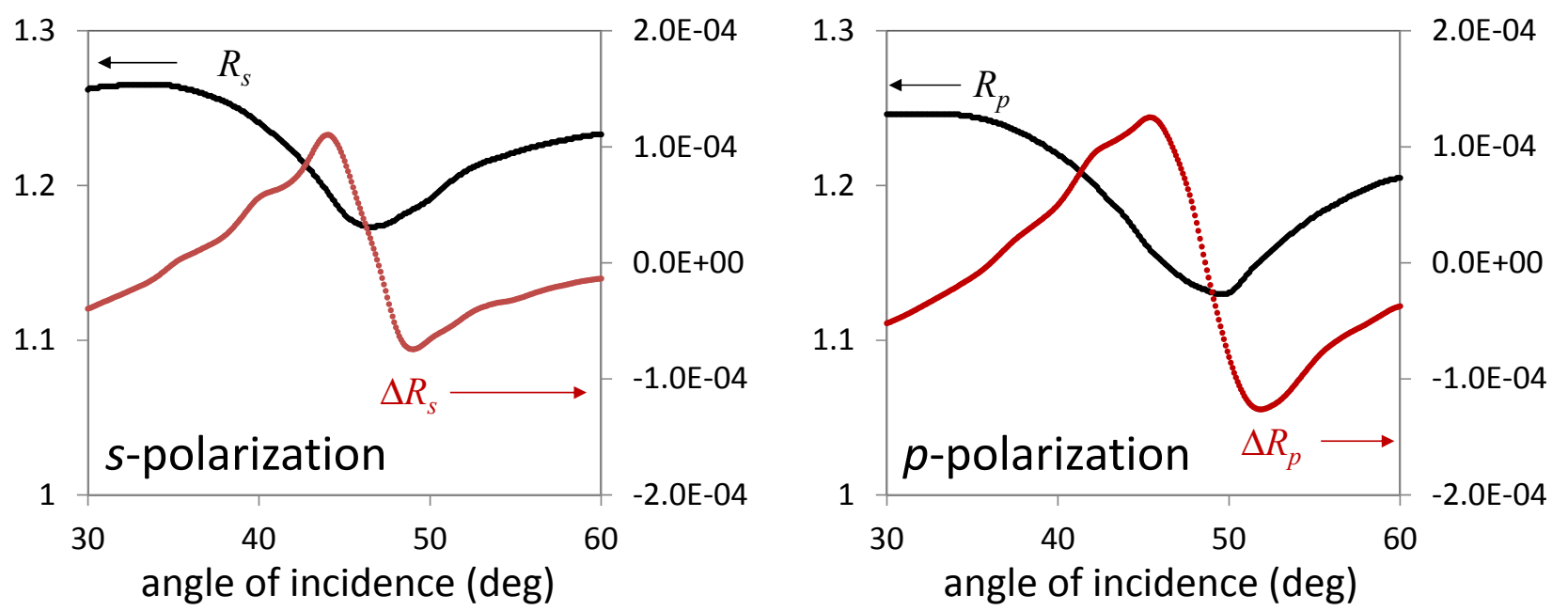

The poled anisotropic indices and thickness can be obtained precisely using the prism coupling technique. In our case, the gold layer was subsequently etched in order to perform an attenuated total reflection (ATR) experiment using a modified prism coupling setup [10] for verification of results from the simplified FP method as well as to obtain measurement of the thickness and anisotropic refractive indices. We obtained $15 \mathrm{pm} / \mathrm{V}$ and $50 \mathrm{pm} / \mathrm{V}$ for $r_{13}$ and $r_{33}$, respectively, using the simplified FP analysis, which showed good agreement (within $~ 5 \%$ ) with the values of $16 \mathrm{pm} / \mathrm{V}$ and $52 \mathrm{pm} / \mathrm{V}$ for $r_{13}$ and $r_{33}$ obtained using the ATR method.

\section{Conclusions}

We have presented a simplified reflection-mode Fabry-Perot method for estimation of EO coefficients of poled polymer thin films using a highly reflective transparent electrode layer. In the simple Teng-Man reflective method [2,3], a highly reflective electrode layer can produce FP effects at infrared wavelengths and result in large errors in the EO coefficients. Here, on the other hand, we have shown that we can utilize enhanced FP effects and typical NLOP samples that are incorporated with the highly reflective electrode layer such as gold or highly absorbing ITO are found to be able to give a reliable estimation of EO coefficients $r_{13}$ and $r_{33}$ separately within an error of $8-10 \%$ without a complete analysis of the multilayer structure. Rigorous analysis of the multilayer structure based on Equations (13) and (14) and optical properties of transparent electrode layer (TCO or thin metal) are not necessary for the analysis. However, accurate measurement of film thickness and anisotropic indices are important to avoid additional uncertainty in the EO coefficients. The $30 \mathrm{wt} . \%$ AJLZ53 in APC host poled under a relatively low poling field of $50 \mathrm{~V} / \mathrm{m}$ showed an $r_{33}$ of $\sim 50 \mathrm{pm} / \mathrm{V}$ at $1,550 \mathrm{~nm}$. The EO response of the chromophore AJLZ53 yielded higher $r_{33}$ values measured independently elsewhere [7] under a higher poling field of $100 \mathrm{~V} / \mathrm{m}$. Finally we expect that the simplified FP analysis combined with a highly reflective transparent conducting layer will be an advantage for polymer synthetic efforts requiring quick and reliable screening of new nonlinear polymer materials. 


\section{Acknowledgements}

Dong Hun Park and Warren N. Herman thank Victor Yun for assistance in construction of a Fabry-Perot experimental setup.

\section{References}

1. Lindsay, G.A.; Singer, K.D. Polymers for second-order nonlinear optics. ACS Symp. Ser. 1995, doi: 10.1021/bk-1995-0601.fw001.

2. Teng, C.C.; Man, H.T. Simple reflection technique for measuring the electro-optic coefficient of poled polymers. Appl. Phys. Lett.1990, 56, 1734-1736.

3. Schildkraut, J.S. Determination of the electro optic coefficient of a poled polymer film. App. Opt. 1990, 29, 2839-2841.

4. Park, D.H.; Lee, C.H.; Herman, W.N. Analysis of multiple reflection effects in reflective measurements of electro-optic coefficients of poled polymers in multilayer structures. Opt. Express 2006, 14, 8866-8884.

5. Michelotti, F.; Belardini, A.; Larciprete, M.C.; Bertolotti, M.; Rousseau, A.; Ratsimihety, A.; Schoer, G.; Mueller, J. Measurement of the electro-optic properties of poled polymers at $\lambda=1.55 \mathrm{~mm}$ by means of sandwich structures with zinc oxide transparent electrode. Appl. Phys. Lett. 2003, 83, 4477-4479.

6. Norwood, R.A.; Kuzyk, M.G.; Keosian, R.A. Electro-optic tensor ratio determination of side-chain copolymers with electro-optic interferometry. J. Appl. Phys. 1994, 75, 1869-1874.

7. Greenlee, C.; Guilmo, A.; Opadeyi, A.; Himmelhuber, R.; Norwood, R.A.; Fallahi, N.; Luo, J.; Huang, S.; Zhou, X.-H.; Jen, A.K.-Y.; Peyghambarian, N. Mach-Zehnder interferometry method for decoupling electro-optic and piezoelectric effects in poled polymer films. Appl. Phys. Lett. 2010, 97, 041109-041109-3.

8. Shi, W.; Ding, Y.J.; Mu, X.; Yin, X.; Fang, C. Electro-optic and electromechanical properties of poled polymer thin films. Appl. Phys. Lett. 2001, 79, 3749-3751.

9. Chen, A.; Chuyanov, V.; Garner, S.; Steier, W.H.; Dalton, L.R. Modified attenuated total reflection for the fast and routine electro-optic measurement of nonlinear optical polymer thin films. Tech. Dig. Org. Thin Films Photonics Appl. 1997, 14, 158-160.

10. Park, D.H. Characterization of Linear Electro-Optic Effect of Poled Organic Thin Films. Ph.D. thesis, University of Maryland, College Park, MD, USA, 2008.

11. Uchiki, H.; Kobayashi, T. New determination method of electro-optic constants and relevant nonlinear susceptibilities and its application to doped polymer. J. Appl. Phys. 1988, 64, 2625-2629.

12. Eldering, C.A.; Knoesen, A.; Kowel, S.T. Use of Fabry-Perot devices for the characterization of polymeric electrooptic films. J. Appl. Phys. 1991, 69, 3676-3686.

13. O’Brien, N.F.; Dominica, V.; Caracci, S. Electro-refraction and electro-absorption in poled polymer Fabry-Perot etalons. J. Appl. Phys. 1996, 79, 7493-7500.

14. Prêtre, Ph.; Wu, L.-M.; Hill, R.A.; Knoesen, A. Characterization of electro-optic polymer films by use of decal-deposited reflection Fabry-Perot microcavities. J. Opt. Soc. Am. B 1998, 15, 379-392. 
15. Yeh, P. Optical Waves in Layered Media; John Wiley \& Sons: Hoboken, NJ, USA, 1988.

16. Rakić, A.D.; Djurišić, A.B.; Elazar, J.M.; Majewski, M.L. Optical properties of metallic films for vertical-cavity optoelectronic devices. Appl. Opt. 1998, 37, 5271-5283.

17. Born, M.; Wolf, E. Principles of Optics, 7th ed.; Cambridge University Press: Cambridge, UK, 1999.

\section{Appendix}

Here, we describe a technique to determine the inverse piezoelectric coefficient of a poled polymer thin film when two or more reflection minima are observed in a reflectivity curve. Taking the derivative of Equation (5) gives

$$
\Delta n_{\gamma}=\frac{\sin 2 \theta}{2 n_{\gamma}} \Delta \theta-\frac{\Delta d}{n_{\gamma} d}\left(n_{\gamma}^{2}-\sin ^{2} \theta\right)
$$

where $\Delta d$ is the change of thickness from the inverse piezoelectric effect. Assuming that two reflection minima in a reflectivity curve exist and that the measured angular shifts are $\Delta \theta_{1}$ and $\Delta \theta_{2}$, we obtain

$$
\left(\begin{array}{c}
\Delta \theta_{1} \\
\Delta \theta_{2}
\end{array}\right)=\left(\begin{array}{cc}
\frac{2 n_{\gamma}}{\sin 2 \theta_{1}} & \frac{2\left(n_{\gamma}^{2}-\sin ^{2} \theta_{1}\right)}{d \sin 2 \theta_{1}} \\
\frac{2 n_{\gamma}}{\sin 2 \theta_{2}} & \frac{2\left(n_{\gamma}^{2}-\sin ^{2} \theta_{2}\right)}{d \sin 2 \theta_{2}}
\end{array}\right)\left(\begin{array}{c}
\Delta n_{\gamma} \\
\Delta d
\end{array}\right)
$$

Using the inverse of this $2 \times 2$ matrix gives

$$
\left(\begin{array}{c}
\Delta n_{\gamma} \\
\Delta d
\end{array}\right)=\left(\begin{array}{cc}
\frac{\sin 2 \theta_{1}\left(n_{\gamma}^{2}-\sin ^{2} \theta_{2}\right)}{2 n_{\gamma}\left(\sin ^{2} \theta_{1}-\sin ^{2} \theta_{2}\right)} & -\frac{\sin 2 \theta_{2}\left(n_{\gamma}^{2}-\sin ^{2} \theta_{1}\right)}{2 n_{\gamma}\left(\sin ^{2} \theta_{1}-\sin ^{2} \theta_{2}\right)} \\
-\frac{d \sin 2 \theta_{1}}{2 n_{\gamma}\left(\sin ^{2} \theta_{1}-\sin ^{2} \theta_{2}\right)} & \frac{d \sin 2 \theta_{2}}{2 n_{\gamma}\left(\sin ^{2} \theta_{1}-\sin ^{2} \theta_{2}\right)}
\end{array}\right)\left(\begin{array}{l}
\Delta \theta_{1} \\
\Delta \theta_{2}
\end{array}\right)
$$

where $\Delta d=d_{33} V$. When only one reflection minimum is observed, one can use a shorter wavelength laser to obtain two or more reflection minima and measure the longitudinal piezoelectric coefficient $d_{33}$ using Equation (17). The two angles can be selected to be as far apart as possible in order to increase the stability of the $2 \times 2$ matrix. Care must be taken to select the shorter wavelength to be outside the absorption band of the polymer thin film, because large absorption causes broadening of the reflectivity and lowering the Q factor as we pointed out in Section 2.

(C) 2011 by the authors; licensee MDPI, Basel, Switzerland. This article is an open access article distributed under the terms and conditions of the Creative Commons Attribution license (http://creativecommons.org/licenses/by/3.0/). 